\title{
Friction tensor concept for textured surfaces
}

\author{
K R Y SIMHA $^{1 *}$, ANIRUDHAN POTTIRAYIL ${ }^{1}$, \\ PRADEEP L MENEZES ${ }^{2}$ and SATISH V KAILAS ${ }^{1}$ \\ ${ }^{1}$ Department of Mechanical Engineering, Indian Institute of Science, \\ Bangalore 560012 \\ ${ }^{2}$ Department of Materials Engineering, Indian Institute of Science, \\ Bangalore 560012 \\ e-mail: simha@mecheng.iisc.ernet.in
}

\begin{abstract}
Directionality of grinding marks influences the coefficient of friction during sliding. Depending on the sliding direction the coefficient of friction varies between maximum and minimum for textured surfaces. For random surfaces without any texture the friction coefficient becomes independent of the sliding direction. This paper proposes the concept of a friction tensor analogous to the heat conduction tensor in anisotropic media. This implies that there exists two principal friction coefficients $\mu_{1,2}$ analogous to the principal conductivities $k_{1,2}$. For symmetrically textured surfaces the principal directions are orthogonal with atleast one plane of symmetry. However, in the case of polished single crystalline solids in relative sliding motion, crystallographic texture controls the friction tensor.
\end{abstract}

Keywords. Friction tensor; surface texture; backward and forward sliding; friction Mohr circle.

\section{Introduction}

It is well known that engineered surfaces exhibit different types of texture. Consequently, the coefficient of friction, $\mu$, need not necessarily be uniform in all directions; or, exhibit the same trend at all points on a surface. Thus, in general, the coefficient of friction behaves like a pseudo tensor function of surface coordinates $\mu_{i j}(x, y)$. However, it is more meaningful to represent this tensor as a function of the direction of sliding. The definition of $\mu_{i j}$ requires a Representative Area Element (RAE) which in the limiting case of RAE $\rightarrow 0$, the friction tensor tends towards a point function. In reality, the friction tensor is a random function which is everywhere continuous but nowhere differentiable. It is therefore strictly not possible to define the friction tensor at a point although the concept is appealing. The situation is somewhat similar to the calculation of the stress tensor in a granular medium. Recently, Menezes et al (2006a,b) studied the effect of roughness parameters and grinding angle on friction coefficients of $\mathrm{Mg}$ and $\mathrm{Al}-\mathrm{Mg}$ alloy pins sliding over EN8 steel flats. The sliding angle was varied between 0 and 90 degrees and morphology of the transfer layer was revealed

${ }^{*}$ For correspondence 
using a scanning electron microscope (SEM). They were able to separate the adhesive and plowing components constituting the friction coefficients a concept dating back to Bowden \& Tabor (1954). It is interesting that Menezes et al noted different coefficients of friction along forward and backward sliding motion due to formation of a transfer layer. Rymuza (1996) has explained friction based on energy dissipation mechanisms initiated in the early history of tribology by Rabinowicz (1951). Despite the spatio-temporal complexity in quantifying surface texture underlying friction, mathematical models for anisotropic friction have been exhaustively discussed by Zmitrowicz $(1981,2003)$.

From the above discussion it is evident that there are various surface/subsurface mechanisms contributing to the directional variation of the coefficient of friction. The heat generated during sliding is mainly conducted into the bulk of the sliding solids. The rate at which heat is conducted away controls the regime of stable sliding for which the definition of the coefficient of friction is meaningful. In cases of heat concentration and hot spot formation, seizures may occur in dry sliding. Seizures may also occur owing to mechanical interlocking of asperities in deeply textured surfaces. It is therefore necessary to provide an appreciation of conduction heat transfer in anisotropic media.

\section{Anisotropic heat conduction}

It is reported in the literature (Bowden \& Tabor 1954; Gahr 1987), that the heat generation influences the coefficient of friction. The rise in temperature during sliding depends on several parameters such as coefficient of friction, sliding velocity, normal load, hardness and thermal conductivity. The flash temperature, $T$, at the frictional contact given by Bowden $\&$ Tabor (1954)

$$
T=\mu \nu f^{\frac{1}{2}} \frac{(\pi h)^{\frac{1}{2}}}{8 k}
$$

where $\mu$ is the coefficient of friction, $v$ the sliding velocity, $f$ the normal load, $h$ the hardness and, finally, $k$ is the thermal conductivity. The thermal conductivity $k$ is assumed to be isotropic in the above equation. In textured media, this assumption is not valid (Eckert \& Drake 1972). The heat flux given by the Fourier law, $q=-k \operatorname{grad} T$, allows for anisotropic thermal conductivity, $k_{i j}$ which forms the connecting link to the friction tensor $\mu_{i j}$. Consider the problem of conduction heat transfer away from the sliding direction, as shown in figure 1.

The sliding direction makes an angle $\beta$ with respect to the principal direction of the texturecontrolled thermal conductivity tensor. The isotherms $\mathrm{AB}$ and $\mathrm{CD}$ are parallel to the sliding direction. The maximum temperature is along the centerline of the slider. Conduction of heat depends on the value of $\beta$. The best conduction occurs along $\beta=0^{\circ}$ while conduction is poor across $\beta=90^{\circ}$. The conduction efficiency is in between the two extreme cases for intermediate angles $0^{\circ}<\beta<90^{\circ}$. The anisotropic heat conduction is given by the equation

$$
\left[\begin{array}{l}
q_{x} \\
q_{y}
\end{array}\right]=\left[\begin{array}{cc}
k_{x} & k_{x y} \\
k_{y x} & k_{y}
\end{array}\right]\left[\begin{array}{c}
\frac{\partial T}{\partial x} \\
\frac{\partial T}{\partial y}
\end{array}\right],
$$

where $q_{x}$ and $q_{y}$ are the heat flux vectors along the $x$ and $y$ directions. The conductivity tensor components $k_{i j}$ are expressed in terms of the principal values $k_{1,2}$ as follows:

$$
k_{x}=k_{1} \cos ^{2} \beta+k_{2} \sin ^{2} \beta
$$




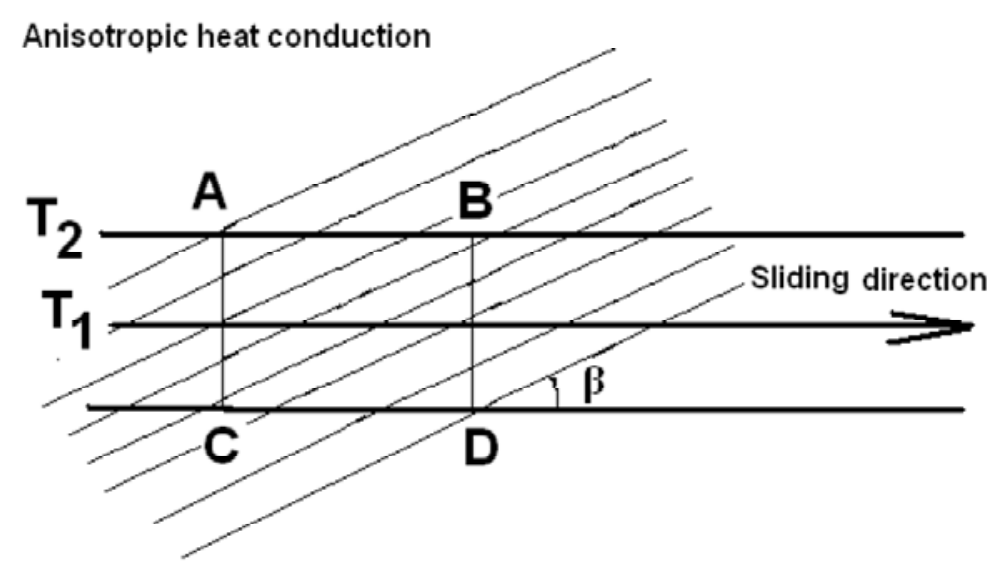

Figure 1. Heat conduction in anisotropic media.

$$
\begin{aligned}
k_{y} & =k_{1} \sin ^{2} \beta+k_{2} \cos ^{2} \beta \\
k_{x y} & =\left(k_{1}-k_{2}\right) \sin (2 \beta) .
\end{aligned}
$$

The heat generated by friction can be modelled as a moving heat source problem in unsteady heat conduction. The unsteady heat conduction equation in a solid of density $\rho$ and specific heat $c$ is

$$
\rho c \frac{\partial T}{\partial t}=k_{x} \frac{\partial^{2} T}{\partial x^{2}}+k_{y} \frac{\partial^{2} T}{\partial y^{2}}+k_{x y} \frac{\partial^{2} T}{\partial x \partial y} .
$$

From the above discussion, we assume that the heat conduction is restricted to a thin active layer engulfing all the asperities, as shown in figure 2.

The variation of $\mu$ with respect to the sliding direction can be represented using circles as in the case of Mohr circle, as shown in figure 3. There are two Mohr circles corresponding to forward sliding and backward sliding, respectively. In this illustration, the forward sliding is smoother than backward sliding indicating that the asperities are tilting in the forward direction. Equivalently, this implies that the slope of the asperities in the backward direction is more than the forward direction, as shown for the texture in figure 3.

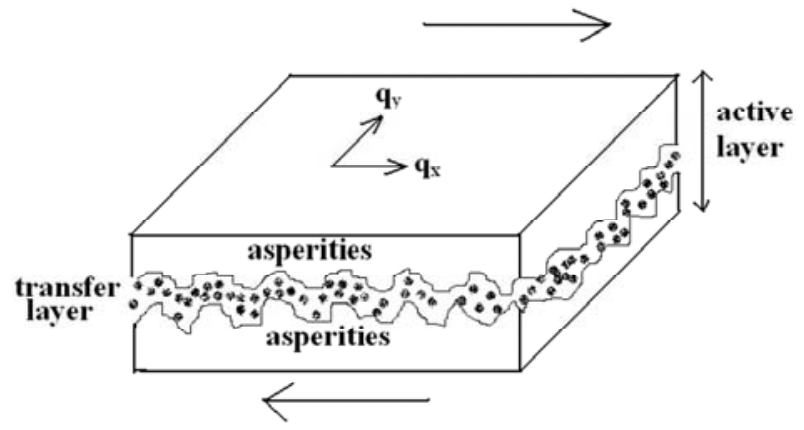

Figure 2. Active layer for heat conduction. 


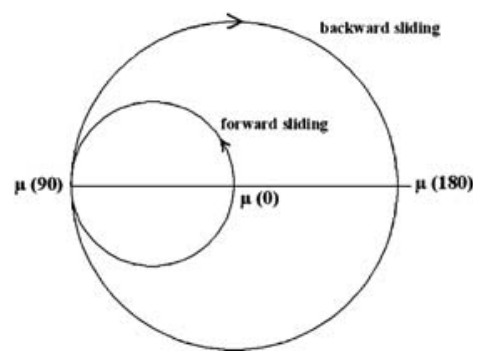

(a)

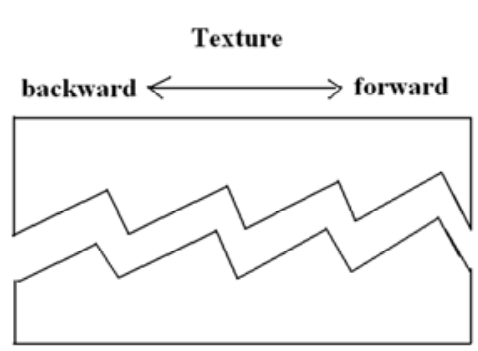

(b)

Figure 3. Friction Mohr circles (a) for texture shown in (b).

\section{Conclusions}

Anisotropic friction resulting from surface texture has been observed experimentally for length scales ranging from few nanometers to tens of microns. This paper makes a proposal for introducing the friction tensor concept for modelling anisotropic heat conduction in textured solids. It is interesting to mention here that the anisotropic effect has been also observed in nano-tribology (Bhushan 2004). Novel methods for measuring temperature effects have become currently available (Farris et al 2003). The random evolution of texture caused by anisotropic friction and wear demands further experimental work to substantiate the ideas proposed here and several other interesting proposals by Zmitrowicz (1995).

\section{References}

Bhushan B 2004 Handbook of Nanotribology. Springer p 642

Bowden F P, Tabor D 1954 The friction and lubrication of solids. Oxford, Clarendon, UK

Eckert E R G, Drake R M 1972 Analysis of heat and mass transfer. (New York: McGraw-Hill Book Company)

Farris T N, Murthy H, Matlik J F 2003 Fretting Fatigue, In R O Rithcie and Y Murakami, Eds., Comprehensive structural integrity Elsevier, Pergamon Press 4: 281-326

Gahr K H Z 1987 Microstructure and wear of materials, Tribology Series, vol 10. (Amsterdam: Elsevier)

Menezes P L, Kishore, Kailas S V 2006a Effect of directionality of unidirectional grinding marks on friction and transfer layer formation of $\mathrm{Mg}$ on steel using inclined scratch test. Mater. Sci. and Eng. A429(1-2): 149-160

Menezes P L, Kishore, Kailas S V 2006b Effect of roughness parameter and grinding angle on coefficient of friction when sliding of Al-Mg alloy over EN8 steel. Trans. ASME: J. Tribol. 128(4): 697-704

Rabinowicz E 1951 Nature of the static and kinetic coefficients of friction. J. Appl. Phys. 22: $1373-1378$

Rymuza Z 1996 Energy concept of the coefficient of friction. Wear 199(2): 187-196

Zmitrowicz A 1981 A theoretical model of anisotropic dry friction. Wear 73: 9-39

Zmitrowicz A 1995 Constitutive models for anisotropic frictional heat. Int. J. Heat Mass Transfer 38(3): 563-574

Zmitrowicz A 2003 Glaciers and laws of friction and sliding. Acta Mech. 166: 185-206 